\title{
ICA injury in sphenoid sinus during nasal endoscopic sinus surgery-a case report
}

\begin{abstract}
Background: Injury to the cavernous portion of the Internal Carotid Artery (ICA) during nasal endoscopic sinus surgery is a rare complication, which is associated with high rates of morbidity and mortality. ICA is more vulnerable to iatrogenic injury inside the sphenoid lateral wall when it runs in a more medial course which although is uncommon but a normal variant and when the ICA runs only in sphenoid sinus mucosal cover.
\end{abstract}

Keywords: carotid artery injury, endoscopic endonasal skull base surgery, functional endoscopic sinus surgery, internal carotid artery, sphenoid sinus, sphenoidotomy, angiography
Volume 5 Issue 2 - 2016

Inderdeep Singh, Sunil Goyal, Sunita Bisht

Department of Otolaryngology Command Hospital India

Correspondence: Inderdeep Singh, Department of

Otolaryngology Head \& Neck surgery, Command Hospital

(Southern Command), Pune, India, Tel +91-7767834137,

Email dridsingh@hotmail.com

Received: August 02, 2016 | Published: December 08, 2016

\section{Introduction}

ICA is a unique in presenting the number of curvatures in different parts of its course and its importance in supplying the brain circulation. It occasionally has one or two flexures near the base of the skull, while in its passage through the carotid canal and along the side of the body of the sphenoid bone it describes a double curvature and resembles the letter ' $\mathrm{S}$ ' or cobra hood in a serpentine course. Surgery in the parasellar and paranasal regions is technically challenging because of the complex anatomic relationships between the sphenoid sinus, cavernous sinus, optic nerve, and internal carotid artery. Normal anatomic variations like metalized course or dehiscence of its bony canal and pathological changes can lead to disastrous outcomes including carotid artery injury. According to literature, intraoperative injury to the Internal Carotid Artery (ICA) during Functional Endoscopic Sinus Surgery (FESS) is a rare complication which ranges from $1.3-3.9 \% .^{2}$ The incidence of ICA injury in transsphenoidal skull base surgery is more common than endoscopic sinus surgery. In the event of a traumatic injury to the ICA during FESS, hemorrhage is difficult to control due to limited access to the sphenoid sinus and the visual field becomes quickly obscured due to massive hemorrhage.

Morbidity is high and it can become fatal within minutes. Mortality rates varies from 30 to $40 \% .{ }^{3,4}$ After controlling bleeding, neurological deficits frequently persist which may be temporary or permanent. The ICA following trauma may undergo pseudoaneurysm changes, carotid cavernous fistula, vessel spasm, thrombosis/embolism or intraluminal dissection of its muscular wall. The immediate measures to manage ICA injury includes controlled hypotension, ipsilateral and contralateral neck compression, pressure packing, ICA ligation in neck. Definite surgical repair depends on defect size and site. Using endoscopic techniques to control bleeding is highly challenging due to constricted surgical field and inability of suturing. Pressure application is suggested when the defect is small. Endonasal repair of the defect has been tried with temporalis muscle fascia with good outcomes. ${ }^{5}$ However, in cases of significant bleeding during FESS, balloon occlusion measures, endovascular treatment was suggested as definitive treatment..$^{6-8}$ In this paper, we provide a detailed description of management of ICA injury in a case with aberrant course with a dehiscent bony wall.

\section{Surgical anatomy}

Sphenoid sinus and cavernous portion of the ICA is separated by a thin bone and sometimes overlying bone is dehiscent. Fujii et al., ${ }^{9}$ in their cadaveric study found that, $88 \%$ of 50 carotid arteries in cadavers had a bony thickness less than $0.5 \mathrm{~mm}$, and in $8 \%$ of cases, the bone overlying the internal carotid artery was dehiscent. Vincentelli et al., ${ }^{10}$ studied 116 cadavers and noted that $4 \%$ of cadavers had minimal bone while Kennedy et al., ${ }^{11}$ found that $20 \%$ of postmortem specimens had a dehiscent intrasphenoid carotid artery. Similar results have been reported in studies of CT scan also. Similarly, in a review of 500 consecutive axial CT scans Johnson et al., ${ }^{12}$ observed that $14.4 \%$ radiographs had minimal to no bone between the two structures. Artery may occasionally protrude into sphenoid sinus, especially when there is an over pneumatization in sphenoid base. ${ }^{13}$

\section{Case report}

35year old male, a known case of Allergic Bronchopulmonary Aspergillosis and Sinonasal Polyposis operated twice previously at different institutions presented to our institution with recurrence of sinonasal polyposis and proptosis of left eye. On diagnostic nasal endoscopy, there was extensive sinonasal polyposis involving bilateral nasal cavities with no landmarks present inside the nose due to previous two surgeries. CT PNS was done to see the extent of disease, anatomical landmarks and extent of orbital proptosis. The findings included: Post op status with bilateral uncinectomy, superior, middle and inferior turbinectomy mucosal thickening and polypoidal changes involving the frontal, ethmoid, sphenoidal and supraorbital air cells. There was left sided intra-orbital extension from ipsilateral supraorbital air cells through orbital roof where the bone is not visualized, causing compression of the globe in postero-lateral aspect.

MRI Brain \& PNS revealed sinus mucosal disease is noted in both maxillary, frontal, sphenoid sinuses and supraorbital air cells. The contents of the left supraorbital air cells are causing pressure erosion of the roof of left orbit with protrusion into orbit posterolaterally and causing indentation of the left superior rectus muscle. There is proptosis of the left eye ball. Endoscopic punch biopsy was taken from nasal cavity which revealed non-specific granulation tissue. Patient planned for FESS

\section{Surgical technique}

Intraoperatively, there was extensive sinonasal polyposis involving $\mathrm{B} / \mathrm{L}$ maxillary, frontal, sphenoid and sinuses. No landmarks can be identified. First disease was removed from right side and then from left side. 
Draf 2 was performed on right side with sphenoidotomy and frontal drill out with modified endoscopic Lothrop procedure on left side. During removal of polypoidal lesions with straight forceps from left sphenoid inadvertently iatrogenic injury of ICA (paraclival part) took place as ICA was aberrantly placed towards midline with dehiscent bony wall. This resulted in torrential bleed and the surgeon quickly did compressible packing with a long nasal pack at the injured site. He was aware of the exact site and sphenoid sinus was wide opened.

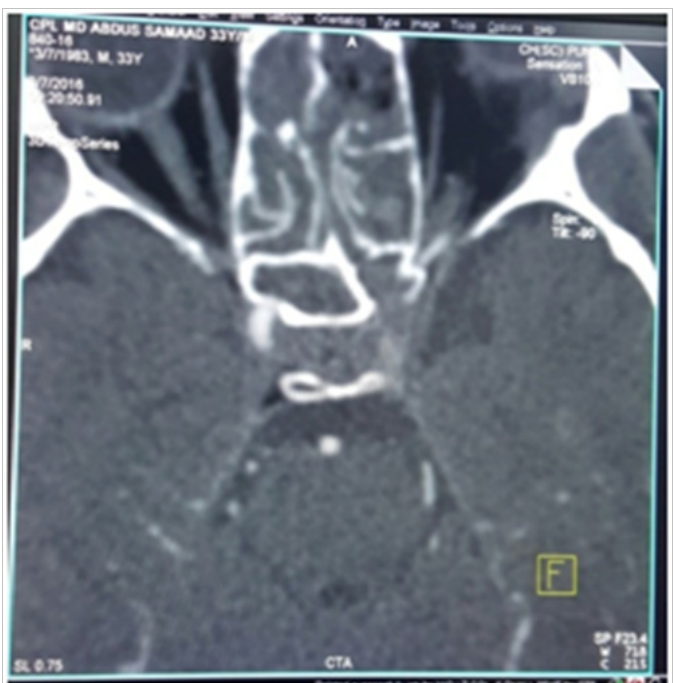

Figure I Immediate post op CT Angiography showing no ischemia or leakage of contrast.

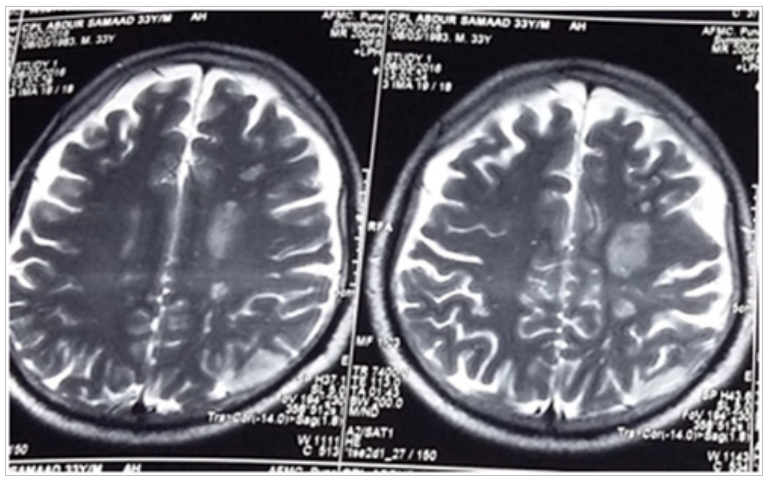

Figure 2 MR Angiography showing ischemic infarct in ACA-MCA \& MCAPCA watershed territory left side.

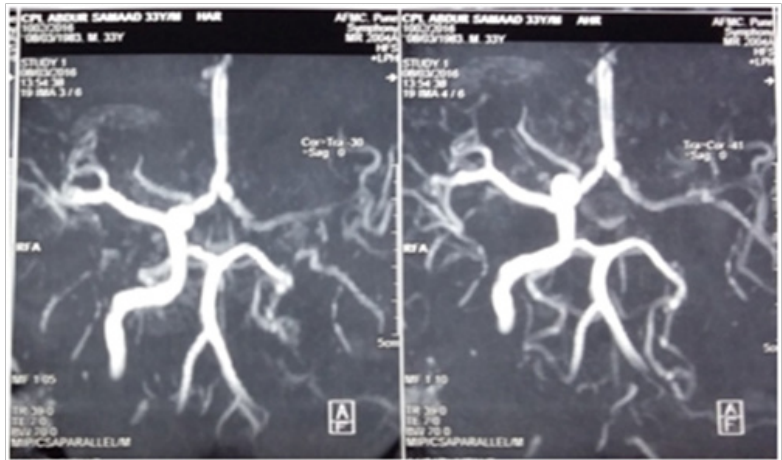

Figure 3 MR Angiogram showing loss of signal in petrous, cavernous and supraclinoid segment of left ICA.

There was $600 \mathrm{ml}$ blood loss. Immediate pressure over left side of neck and nasal packing was carried out with saline soaked gauze which stopped the active bleeding temporarily. Surgery was completed with the clearance of disease at orbital apex with a medial orbital decompression on the left side. After two hours using twosurgeon four-hand technique the pack was removed and there was again a torrential bleed. A definitive packing with a crushed muscle tissue from the temporalis and a surgical combination was put over the injured site for better clotting effect and it was reinforced and compressed with saline soaked long ribbon gauze which stopped the bleeding. Overall blood loss after the completion of surgery was one and half litres. Two points packed cells and fresh frozen plasma was given in the operating room and intervention radiologist was informed for standby. Vital signs were monitored carefully; arterial blood was drawn to monitor blood gases and hematocrit levels.

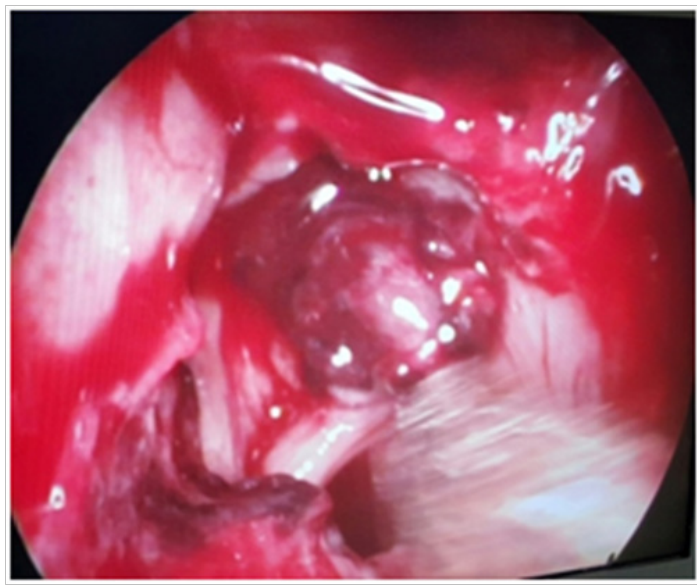

Figure 4 ICA Clot after immediate removal of nasal packing.

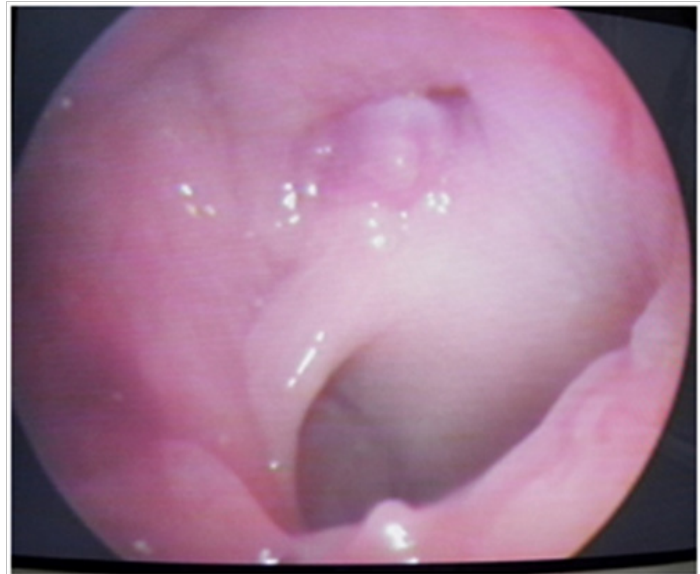

Figure 5 Organised clot 14 postoperative day.

\section{Postoperative management}

After ensuring no active bleeding and patient was hemodynamically stable he was extubated uneventfully and shifted to Intensive care unit. Post op CT Angiography done on same day after three hours revealed acute thrombosis at left distal cervical, petrous, and cavernous ICA. Bony defects/dehiscence has be seen in posterolateral aspect of sphenoid sinus in relation to course of ICA. B/L the gap between midline of sphenoid and the ICA was measuring less than $4 \mathrm{~mm}$ on left and $5 \mathrm{~mm}$ on right side. Left sphenoid was also smaller than the right side. No evidence of early ischemic changes noted in brain parenchyma. No active contrast leak or extravasations seen. There was adequate collateral supply from opposite ICA and circulation 
flow was adequate in circle of Willis and posterior fossa (Figure 1). In view of adequate brain circulation and a localized thrombus in injured ICA the decision was taken in consultation with interventional radiologist and neurologist to manage it conservatively rather opting for any endovascular stent option.

On postoperative day one, pt developed Gd IV weakness (R) side which progressed to Gd III over next 2days. In view of progressing neurological deterioration clinically, low dose ecospirin 150mg OD and LMWH antiplatelet therapy started and intranasal pack removal planned after 48hours in OT under GA.

Before shifting patient to operation theatre, a repeat MR Angiography was done to ensure that there is a stable thrombus at the injury site before pack removal. It showed an ill defined hypodense areas involving gray and white matter in left frontal and parietal lobes in the region of ACA-MCA and MCA-PCA watershed territories respectively, suggestive of watershed territory infracts. Ill defined hypodense areas suggestive of infracts are seen in left centrum semiovale in vicinity of central sulcus (Figure 2) and loss of signal intensity in petrous, cavernous and supraclival part of left ICA (Figure 3). Nasal pack removed under GA with no re-bleeding from injured site. Clot was seen over the injured part of ICA in the left sphenoid sinus plugging the LT ICA (Figure 4). Postoperative antiplatelet therapy continued augmented with physiotherapy.

Patient was mobilized by 3rd postop day with support and intensive physiotherapy was started. By 5 th day he started walking and by the end of a week his Power in limbs of right side improved. Post op regular every day endoscopic suction toileting of nasal cavity and PNS was done carefully so as to avoid any infection or crusts and to assess how clot is organizing over the injured part of Lt ICA in left sphenoid sinus (Figure 5). At 3weeks the clot was fully flushed with bony canal of ICA and almost became indistinguishable. MRI showed improvement in the circulation at the infarct areas. The patient regained all his motor functions by 4 weeks and he could do all normal activities.

The ICA indents the lateral wall of the sphenoid sinus in $71 \%$ to $98 \%$ of cases and may be dehiscent in $4 \%$ to $22 \%$. Fujii and colleagues ${ }^{14}$ noted a $98 \%$ incidence of the ICA bulging into the sphenoid sinus, an $8 \%$ incidence of bony dehiscence, and an $88 \%$ incidence of bone less than $0.5 \mathrm{~mm}$ covering the artery. Gardner et al., ${ }^{15} \mathrm{~m}$ in their study of 2015 endonasal skull base surgeries, encountered 7 ICA injuries in giving an incidence of $0.3 \%$. Most injuries (5 of 7) involved left ICA, and most common diagnosis was chondroid neoplasm (3 of 7). The paraclival ICA segment was most commonly injured site (5 of 7), and transclival/transpteroid approaches had a higher incidence of injury. ICA injury during endonasal skull base surgery is an infrequent and manageable complication. Carotid arterial injuries have potential for devastating complications. Published studies report $23 \%$ to $28 \%$ mortality rates, with $48 \%$ to $58 \%$ of survivors having permanent severe neurologic deficits. ${ }^{9}$

Therefore, when such a life threatening complication occurs, prompt identification and intervention is crucial. Identification of the ICA is essential when operating in the cavernous region. The posterior wall of the sphenoid sinus, which has the optic and carotid prominences, should not be considered sufficient protection and avoiding injury is the best management. ICA is more prone to injury if it has an aberrant parasphenoidal course or there is dehiscence of bony covering. A retrospective review by Johnson et al., ${ }^{12}$ was made of 500 consecutive thin-section axial CT scans of the adult sphenoid sinus using contrast enhancement and a bone algorithm to determine whether there was exposure of the cavernous sinus and the carotid arteries along their parasphenoidal course was formulated.

At least one cavernous-carotid complex that could be considered at risk during sphenoid sinus surgery was seen in $31.4 \%$ of the patients. Of this group, $14.4 \%$ of the patients had no bony covering on one or both sides at some point along the parasphenoidal course. In $17 \%$ the bony covering was so thin it would give minimal, if any, protection from surgical trauma. A number of techniques have been tested, including muscle patch treatment, Floseal, oxidized regenerated cell, vascular clamps and aneurysm U Clip, anastomotic sutures to seal the vascular defect. Packing and placing adequate pressure has the potential to stop the bleeding, however, applying enough pressure to stop the bleeding can lead to thrombosis and complete occlusion of the artery. Ischemia of the brain may occur as a result of reduced blood flow to the brain. Pressure application can lead to pseudoaneurysm formation, especially following endovascular recanalization. Endovascular techniques remain valid option if endonasal repair is not achieved. Two commonly used endovascular techniques include balloon occlusion and coil embolization, and endovascular stent graft placement.

\section{Conclusion}

The mortality and morbidity of an ICA injury during FESS can be decreased if the team is properly trained in sphenoid sinus surgery and anticipate it in cases where ICA lies without its bony cover in lateral wall of sphenoid mucosa, the distance is $4 \mathrm{~mm}$ or less between ICA and midline of sphenoid sinus especially in small size sphenoid sinus or when intersphenoid bony septum is attached to thin bone over ICA in preoperative computed tomography (CT). This validates the importance of team approach and co-operation with radiologist. It is imperative in revision cases to identify prior surgical changes and anatomical landmarks if present. In event of injury to ICA, management can be done by early recognition of the injury, immediate packing, blood transfusion and repair of the defect if possible. Postoperative anticoagulants has a role after evaluating the extent of thrombosis in postop scans and in cases thrombosis is progressive then endovascular stenting can be considered. Immediate and regular physiotherapy is helpful and the follow up is done with MRI scans including clinical evaluation of neurological functions and with nasal endoscopic examinations. In cases of good posterior circulations and collaterals the recovery is complete.

\section{Acknowledgments}

None.

\section{Conflicts of interest}

Author declares there are no conflicts of interest.

\section{Funding}

None.

\section{References}

1. Drake RL, Vogl W, Mitchell AWM, et al. Gray's Anatomy for Students: Churchill Livingstone/Elsevier, Philadelphia, USA. 2010.

2. Weidenbecher $M$, Huk WJ, Iro H. Internal carotid artery injury during functional endoscopic sinus surgery and its management. Eur Arch Otorhinolaryngol. 2005;262(8):640-645.

3. Perry M, Snyder W, Thal E. Carotid artery injuries caused by blunt trauma. Ann surg. 1980;192(1):74-77. 
4. Fabian TC, Patton JH, Croce MA, et al. Blunt carotid injury Importance of early diagnosis and anticoagulant therapy. Ann surg. 1996;223(5):513-522.

5. Duek I, Svir G, Amit M, et al. Endoscopic Repair of Carotid Artery Injury. Otolaryngol (Sunnyvale). 2015;5:211.

6. Labruzzo SV, Aygun N, Zinreich SJ. Imaging of the Paranasal Sinuses: Mitigation, Identification, and Workup of Functional Endoscopic Surgery Complications. Otolaryngol Clin North Am. 2015;48(5):805-815.

7. Ventureyra E, Higgins M. Traumatic intracranial aneurysms in childhood and adolescence. Child's Nerv Syst. 1994;10(6):361-379.
8. Park AH, Stankiewicz JA, Chow J, et al. A protocol for management of a catastrophic complication of functional endoscopic sinus surgery: internal carotid artery injury. Am J Rhinol. 1998;12(3):153-158.

9. Fujii K, Chambers SM, Rhoton AL. Neurovascular relationships of the sphenoid sinus: a microsurgical study. J Neurosurg. 1979;50(1):31-39.

10. Moore EE, Ryu RK, et al. The unrecognized epidemic of blunt carotid arterial injuries: early diagnosis improves neurologic outcome. Ann Surg. 1998;228(4):462-470. 